Supporting Information

\title{
Electric-field Tunable Magnetism in van der Waals Bilayers with A- type Antiferromagnetic Order: Unipolar versus Bipolar Magnetic
}

\section{Semiconductor}

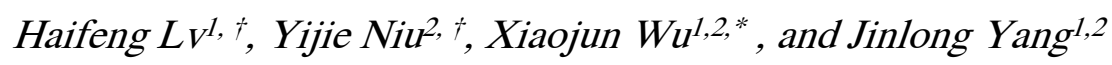

${ }^{1}$ Hefei National Laboratory for Physical Sciences at the Microscale, University of Science and Technology of China, Hefei, Anhui 230026, China.

${ }^{2}$ CAS Key Laboratory for Materials for Energy Conversion, School of Chemistry and Materials

Science, CAS Center for Excellence in Nanoscience and Synergetic Innovation of Quantum Information \& Quantum Technology, University of Science and Technology of China, Hefei, Anhui 230026, China.

Corresponding Author

*E-mail: xjwu@ustc.edu.cn 


\section{Computational details}

First-principles spin-polarized calculations are performed within the density-functional theory (DFT) employing the projected augmented wave (PAW) method, as implemented in the Vienna ab initio simulation package (VASP). ${ }^{1-5}$ Energy cutoff for the plane wave functions is $520 \mathrm{eV}$ and $350 \mathrm{eV}$ for bilayer $\mathrm{Cr}(\mathrm{pyz})_{2}$ and bilayer $\mathrm{NiBi}_{2} \mathrm{Te}_{4} \cdot{ }^{6-8}$ Lattice constants and atomic positions are optimized unless forces on each atom are less than $0.01 \mathrm{eV} / \AA$ and convergence criterion for energy is $1 \times 10^{-6} \mathrm{eV}$. For bilayer $\mathrm{NiBi}_{2} \mathrm{Te}_{4}$ and bilayer $\mathrm{Cr}(\text { pyz })_{2}$, we adopt Hubbard on-site Coulomb parameter $\mathrm{U}_{\text {eff }}$ of $4 \mathrm{eV}$ and $3 \mathrm{eV}$ for $\mathrm{Ni}^{2+}$ and $\mathrm{Cr}^{2+}$ atoms..$^{6-8}$ Van der Waals interaction of bilayer systems is described by DFT-D3 method. ${ }^{9}$ The reduced Brillouin zone is sampled with a $\Gamma$-centered k-grid $12 \times 12 \times 1$ and $6 \times 6 \times 1$ for bilayer $\mathrm{NiBi}_{2} \mathrm{Te}_{4}$ and bilayer $\mathrm{Cr}(\mathrm{pyz})_{2}$. The vacuum regions are set to be above $15 \AA$ for all systems. The phonon band structures are calculated by using PHONOPY code ${ }^{10}$ with the finite displacement method. 

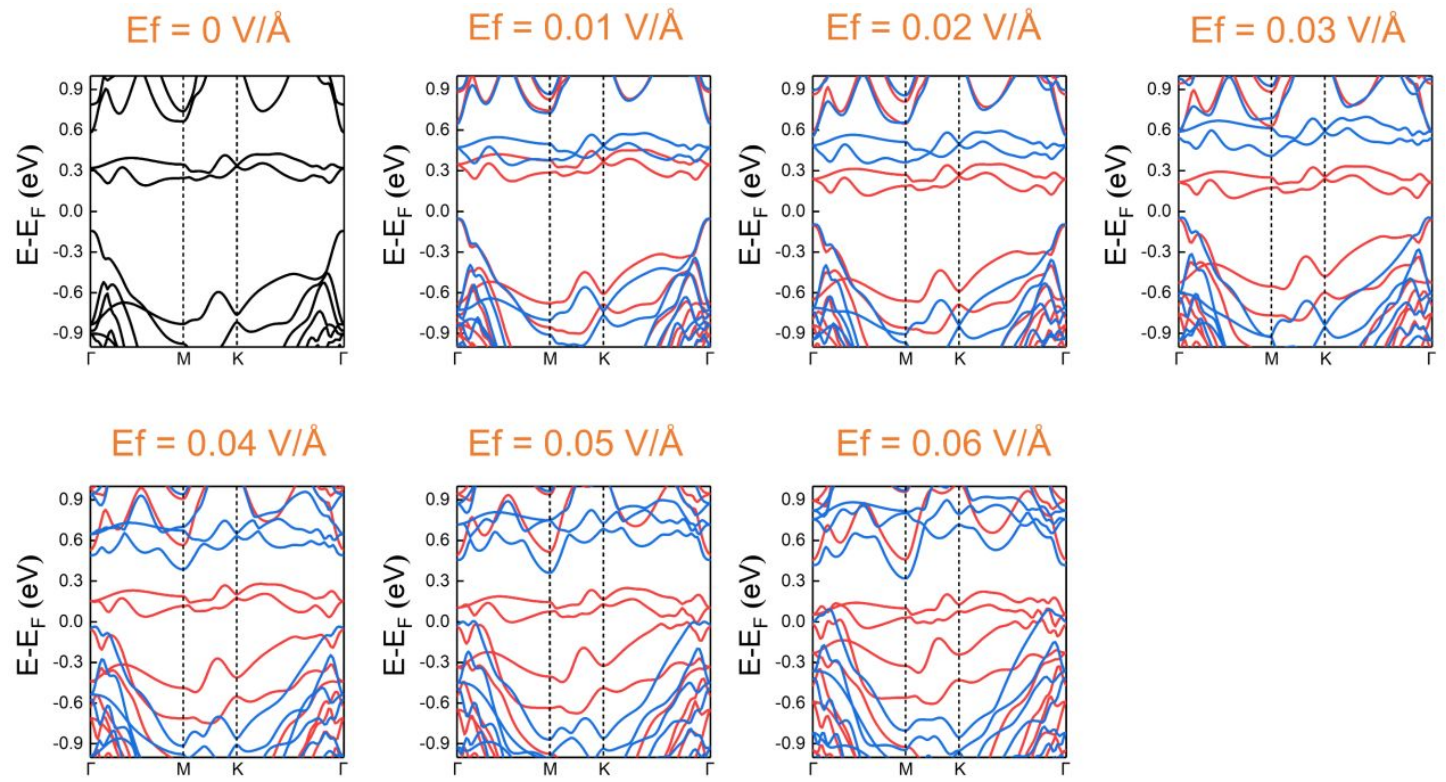

Figure S1. Electronic band structures of bilayer $\mathrm{NiBi}_{2} \mathrm{Te}_{4}$ upon electric fields ( $\mathrm{Ef}$ ) from 0 to 0.06 V/Å. 

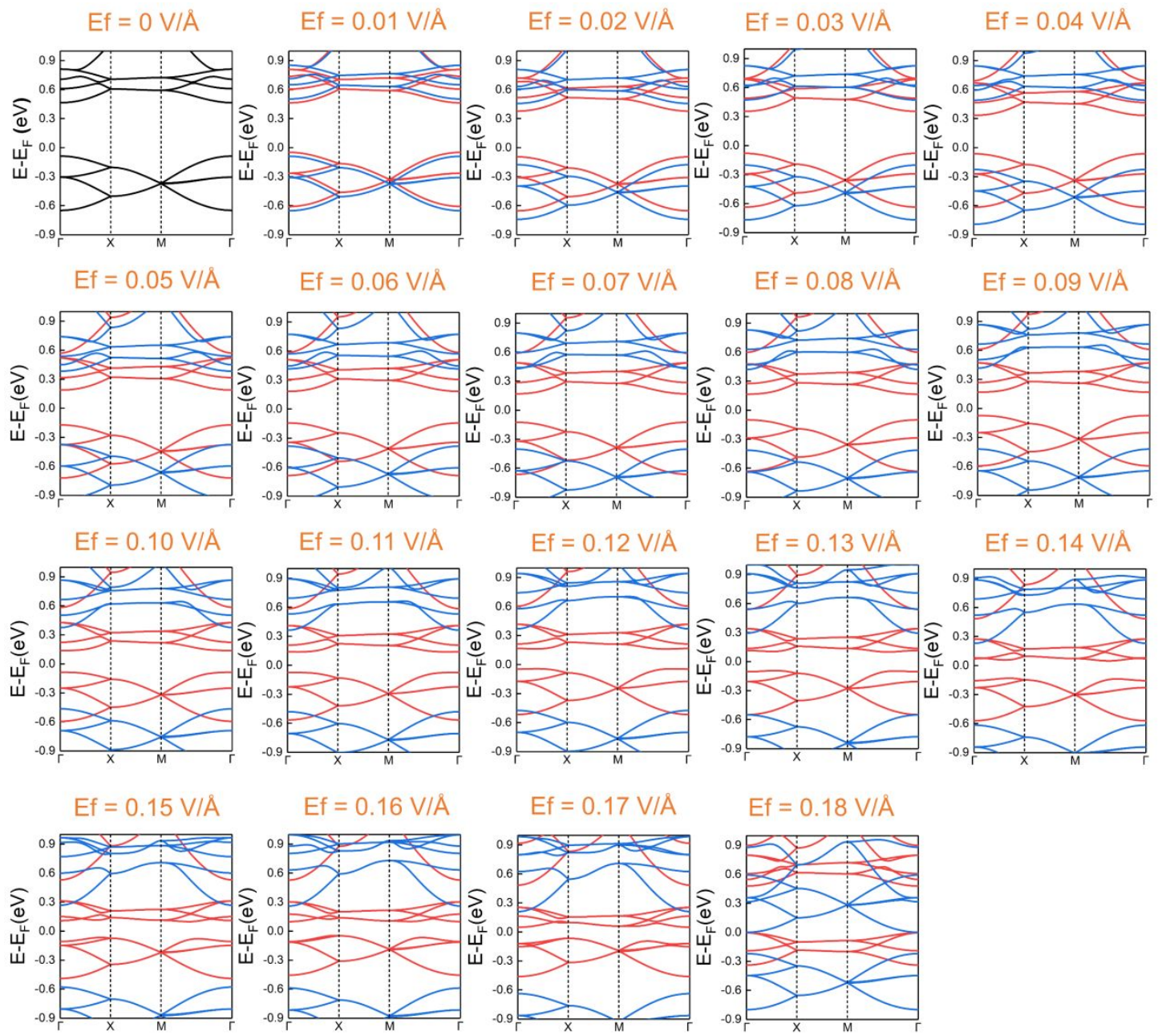

Figure S2. Electronic band structures of bilayer $\mathrm{Cr}(\mathrm{pyz})_{2}$ upon electric fields (Ef) from 0 to 0.18

$\mathrm{V} / \mathrm{A}$ 
a

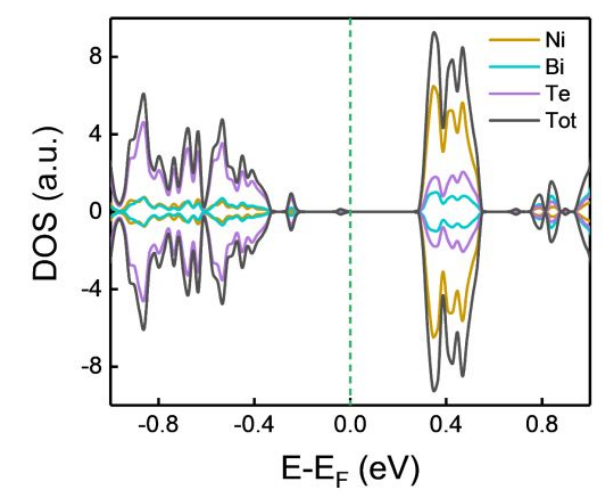

C

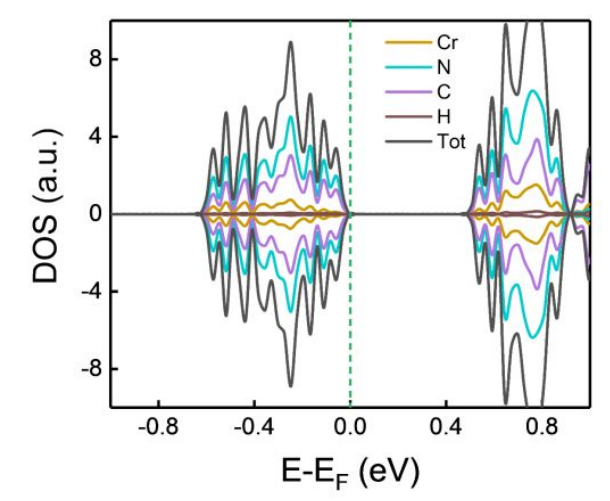

b

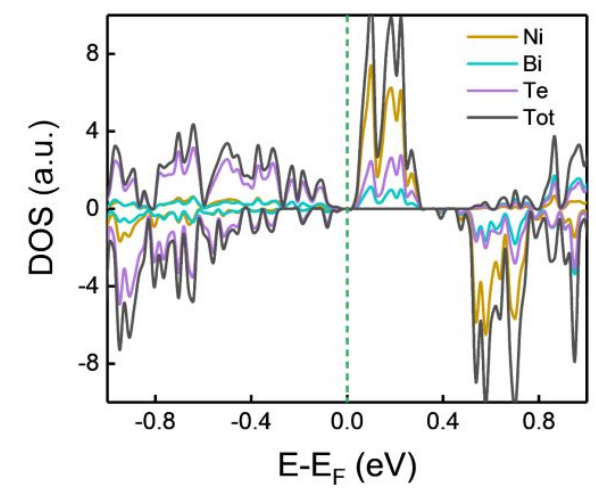

d

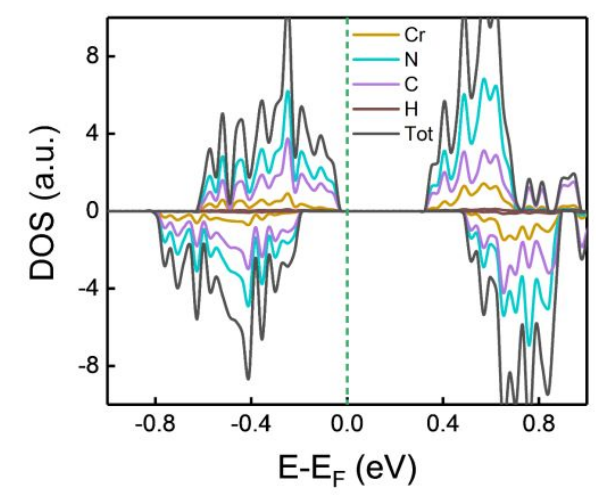

Figure S3. Calculated density of states (DOS). (a-b) $\mathrm{NiBi}_{2} \mathrm{Te}_{4}$ bilayer upon electric field of 0 V/Å and $0.04 \mathrm{~V} / \mathrm{A}$. (c-d) $\mathrm{Cr}(\mathrm{pyz})_{2}$ bilayer upon electric field of $0 \mathrm{~V} / \AA ̊$ and $0.04 \mathrm{~V} / \AA ̊$. 
a
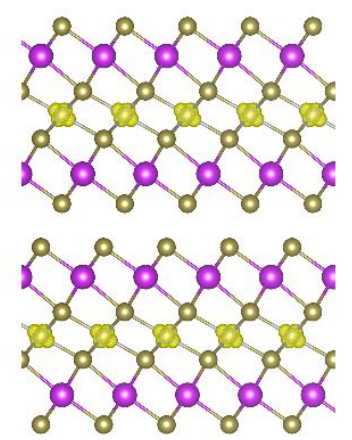

C
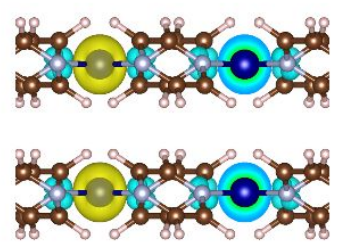

b
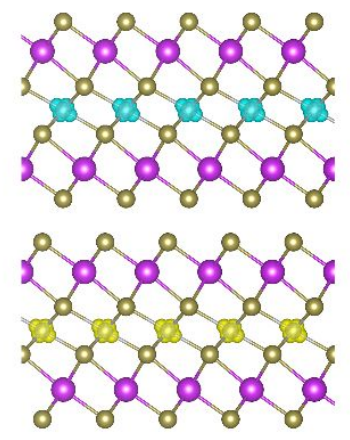

d
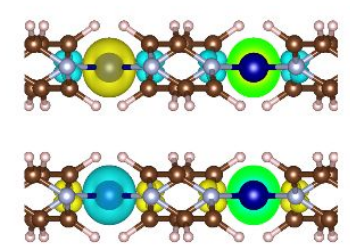

Figure S4. Various interlayer magnetic states for bilayer $\mathrm{NiBi}_{2} \mathrm{Te}_{4}$ and bilayer $\mathrm{Cr}(\mathrm{pyz})_{2}$. (a, c)

ferromagnetic state (FM) and A-type antiferromagnetic state (AFM) for bilayer $\mathrm{NiBi}_{2} \mathrm{Te}_{4} ;(\mathrm{b}, \mathrm{d})$

FM and A-type AFM for bilayer $\mathrm{Cr}(\mathrm{pyz})_{2}$. For bilayer $\mathrm{NiBi}_{2} \mathrm{Te}_{4}$, isosurface levels are set to be $0.015 \mathrm{e} / \mathrm{bohr}^{3}$. For bilayer $\mathrm{Cr}(\mathrm{pyz})_{2}$, isosurface level is set to be $0.03 \mathrm{e} / \mathrm{bohr}^{3}$. 

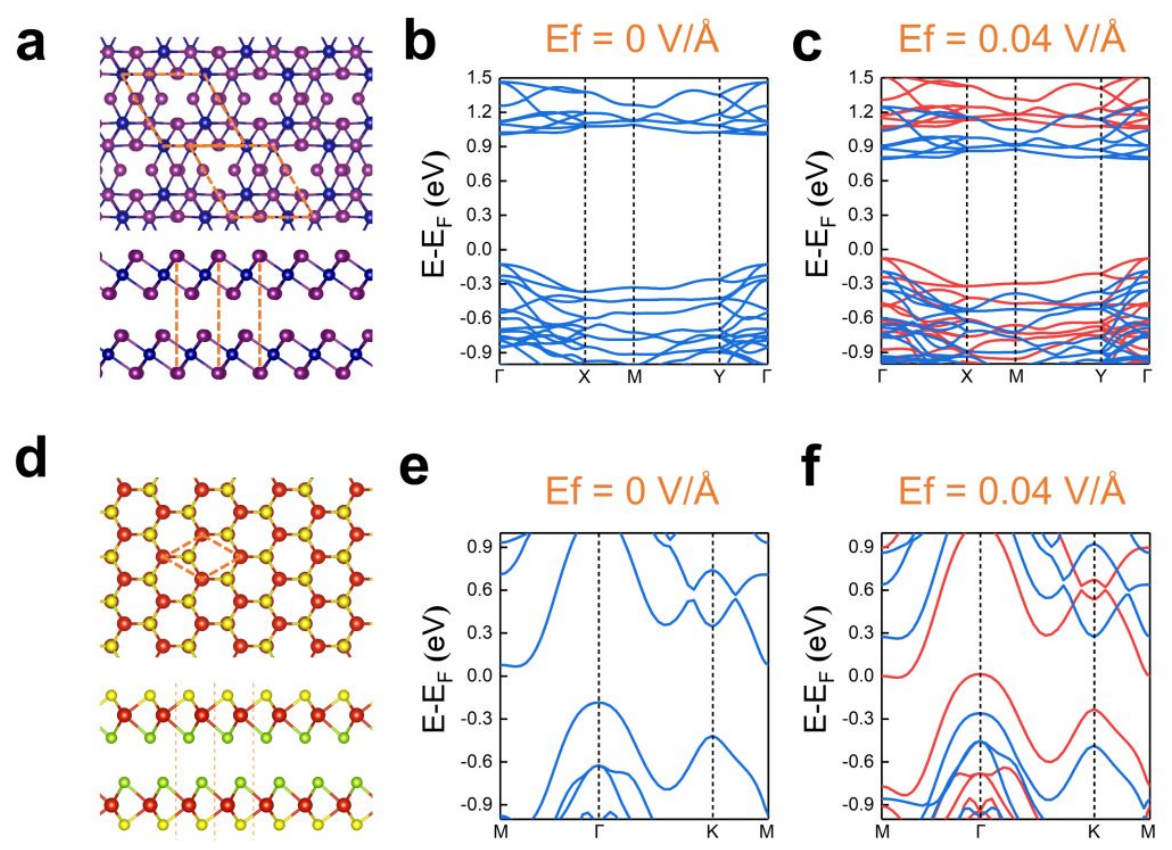

Figure S5. (a, d) Crystal structures of bilayer $\mathrm{CrI}_{3}$ in $\mathrm{AA}^{\prime}$-stacking and bilayer VSSe in A'Astacking in top view and side view; $(b, e)$ Electronic band structures upon zero electric field $(\mathrm{Ef}=$ $0 \mathrm{~V} / \AA ̊ \AA)$ and $(\mathrm{c}, \mathrm{f})$ upon perpendicular electric fields $(\mathrm{Ef}=0.04 \mathrm{~V} / \AA ̊ \AA)$ for bilayer $\mathrm{CrI}_{3}$ and $(\mathrm{Ef}=$ $0.04 \mathrm{~V} / \AA ̊$ ) for bilayer VSSe. Blue and red are denoted as spin- $\alpha$ and spin- $\beta$, respectively. 
a

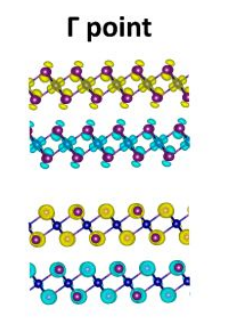

b

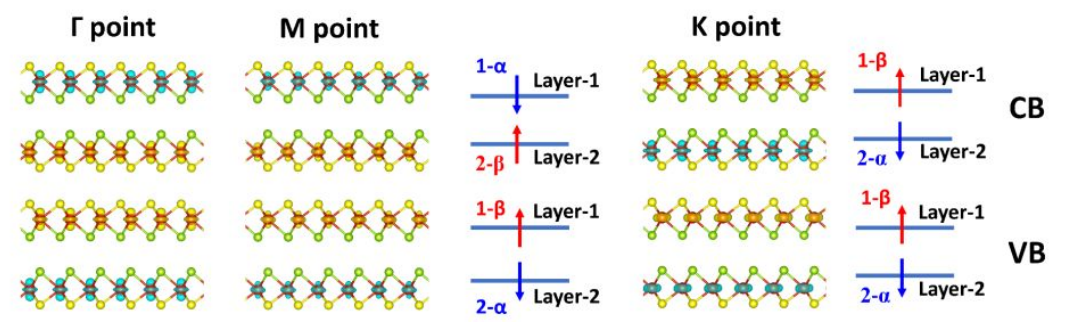

Figure S6. (a) Projected spin density of $\Gamma / \mathrm{M} / \mathrm{X}$ high-symmetry k-points of $\mathrm{CB}$ and VB in real space for bilayer $\mathrm{CrI}_{3}$; (e) Projected spin density of $\Gamma / \mathrm{M} / \mathrm{K}$ high-symmetry k-points of $\mathrm{CB}$ and VB in real space for bilayer 2H-VSSe. Note: For bilayer 2H-VSSe, isosurface levels are set to be $0.005 \mathrm{e} / \mathrm{bohr}^{3}$ (M point) and $0.01 \mathrm{e}^{\mathrm{b}} \mathrm{bohr} \mathrm{r}^{3}$ for $\left(\Gamma\right.$ and $\mathrm{K}$ point), respectively. For bilayer $\mathrm{CrI}_{3}$, isosurface level is set to be $0.001 \mathrm{e} / \mathrm{bohr}^{3}$. 

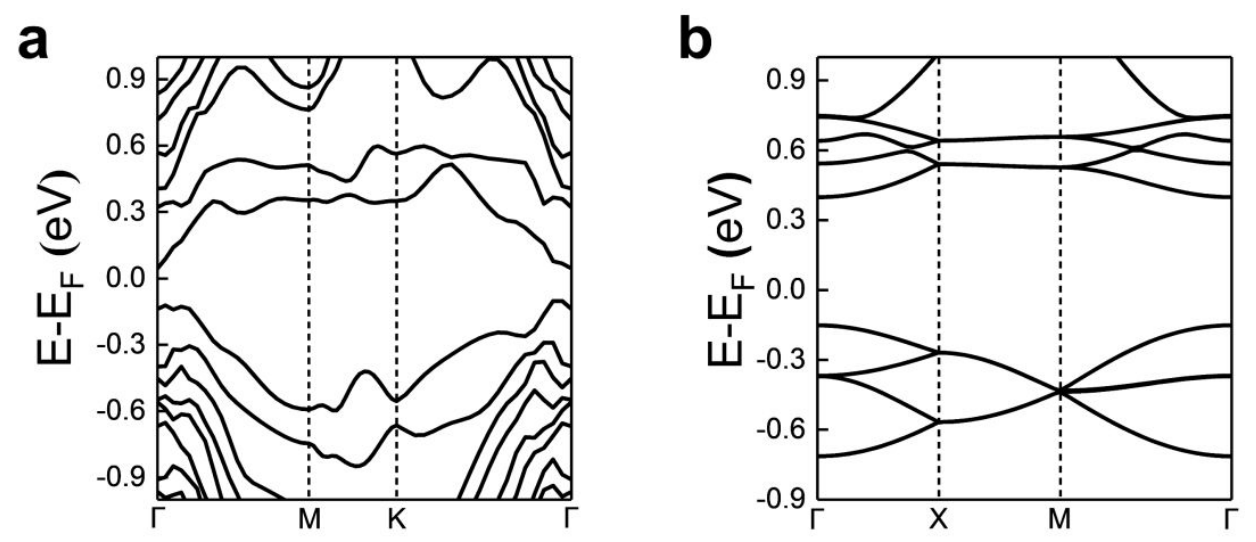

Figure S7. Calculated band structures including spin-orbital coupling effect. (a) $\mathrm{NiBi}_{2} \mathrm{Te}_{4}$ bilayer and (b) $\mathrm{Cr}(\text { pyz })_{2}$ bilayer. 
a

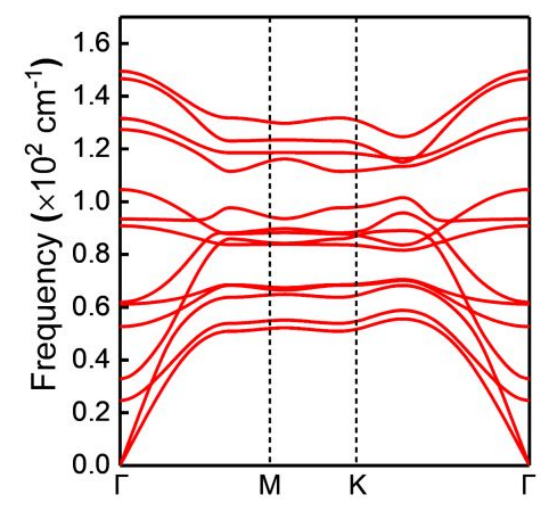

b

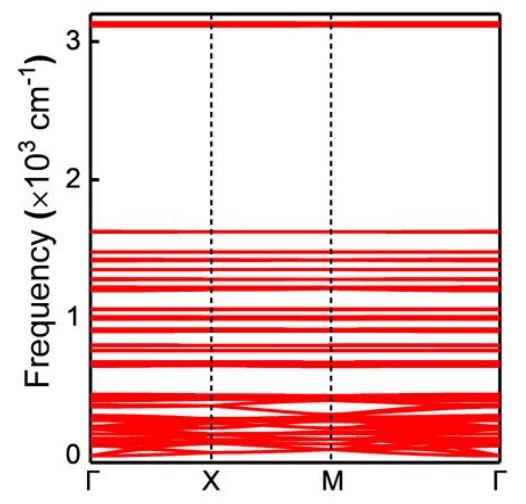

Figure S8. Calculated phonon band structures. (a) $\mathrm{NiBi}_{2} \mathrm{Te}_{4}$ monolayer and (b) $\mathrm{Cr}(\mathrm{pyz})_{2}$ monolayer. The absence of imaginary frequency indicates the lattice stability of $\mathrm{NiBi}_{2} \mathrm{Te}_{4}$ monolayer and $\operatorname{Cr}(\mathrm{pyz})_{2}$ monolayer. 
Table S1. For bilayer $\mathrm{NiBi}_{2} \mathrm{Te}_{4}$ and bilayer $\mathrm{Cr}(\mathrm{pyz})_{2}$, calculated lattice parameters $\boldsymbol{a}$ and $\boldsymbol{b}(\AA)$, interlayer distance $\Delta d_{z}$, electronic bandgap $E_{g}(\mathrm{eV})$, atomic magnetic moment $\boldsymbol{\mu}\left(\mu_{\mathrm{B}}\right)$, energy differences of various magnetic states (AFM: A-type antiferromagnetic state, FM: ferromagnetic state) $E_{F M-A F M}(\mathrm{meV} /$ unit cell), ground state configurations (G.S.).

\begin{tabular}{cccccccc}
\hline \hline Bilayers & $\boldsymbol{a}$ & $\boldsymbol{b}$ & $\boldsymbol{\Delta} \boldsymbol{d}_{\boldsymbol{z}}$ & $\boldsymbol{E}_{\boldsymbol{g}}$ & $\boldsymbol{\mu}$ & $\boldsymbol{E}_{\boldsymbol{F M} \text { - } \boldsymbol{A F M}}$ & $\boldsymbol{G . S .}$ \\
\hline $\mathrm{NiBi}_{2} \mathrm{Te}_{4}$ & 4.300 & 4.300 & 2.564 & 0.344 & 1.275 & 25.517 & AFM \\
$\mathrm{Cr}(\text { pyz })_{2}$ & 9.905 & 9.905 & 1.899 & 0.552 & 3.519 & 61.317 & AFM \\
\hline
\end{tabular}


Table S2. Calculated magnetocrystalline anisotropy energy (MAE) $\mathrm{E}_{[100]}-\mathrm{E}_{[001]}, \mathrm{E}_{[010]}-\mathrm{E}_{[001]}$, $\mathrm{E}_{[110]}-\mathrm{E}_{[001]}$, and $\mathrm{E}_{[111]}-\mathrm{E}_{[001]}(\mu \mathrm{eV} /$ unit cell) and easy magnetization axis EMA. The [001] is the out-of-plane direction.

\begin{tabular}{cccccc}
\hline \hline Bilayers & $\mathrm{E}_{[100]}-\mathrm{E}_{[001]}$ & $\mathrm{E}_{[010]}-\mathrm{E}_{[001]}$ & $\mathrm{E}_{[110]}-\mathrm{E}_{[001]}$ & $\mathrm{E}_{[111]}-\mathrm{E}_{[001]}$ & EMA \\
\hline $\mathrm{NiBi}_{2} \mathrm{Te}_{4}$ & 336.59 & 335.31 & 335.63 & 148.46 & out-of-plane \\
$\mathrm{Cr}(\mathrm{pyz})_{2}$ & 956.40 & 952.96 & 954.85 & 636.67 & out-of-plane \\
\hline \hline
\end{tabular}

\section{REFERENCE}

1 G. Kresse and J. Hafner, Ab initio molecular dynamics for liquid metals. Phys. Rev. B $1993,47,558-561$.

2 P. E. Blöchl, Projector augmented-wave method. Phys. Rev. B 1994, 50, 17953-17979.

3 G. Kresse and J. Furthmüller, Efficient iterative schemes for ab initio total-energy calculations using a plane-wave basis set. Phys. Rev. B 1996, 54, 11169-11186.

4 J. P. Perdew, K. Burke and M. Ernzerhof, Generalized gradient approximation made simple. Phys. Rev. Lett. 1996, 77, 3865-3868.

5 G. Kresse and D. Joubert, From ultrasoft pseudopotentials to the projector augmentedwave method. Phys. Rev. B 1999, 59, 1758-1775. 

magnetic topological insulators in van der Waals layered MnBi2Te4-family materials. Sci. $A d v$. 2019, 5, eaaw5685.

7 P. Perlepe, I. Oyarzabal, A. Mailman, M. Yquel, M. Platunov, I. Dovgaliuk, M. Rouzières, P. Négrier, D. Mondieig, E. A. Suturina, M.-A. Dourges, S. Bonhommeau, R. A. Musgrave, K. S. Pedersen, D. Chernyshov, F. Wilhelm, A. Rogalev, C. Mathonière and R. Clérac, Metal-organic magnets with large coercivity and ordering temperatures up to $242^{\circ} \mathrm{C}$. Science 2020, 370, 587.

8 Z. Li, J. Li, K. He, X. Wan, W. Duan and Y. Xu, Tunable interlayer magnetism and band topology in van der Waals heterostructures of MnBi2Te4-family materials. Phys. Rev. B 2020, 102, 081107.

9 S. Grimme, J. Antony, S. Ehrlich and H. Krieg, A consistent and accurate ab initio parametrization of density functional dispersion correction (DFT-D) for the 94 elements H-Pu. $J$. Chem. Phys. 2010, 132, 154104.

10 A. Togo and I. Tanaka, First principles phonon calculations in materials science. Scr. Mater. 2015, 108, 1-5. 